\title{
Live attenuated varicella-zoster virus vaccine does not induce HIV target cell activation
}

\author{
Catia T. Perciani, ${ }^{1}$ Bashir Farah, ${ }^{2}$ Rupert Kaul, ${ }^{1,3,4}$ Mario A. Ostrowski, ${ }^{1,3,5}$ Salaheddin M. Mahmud, ${ }^{6}$ Omu Anzala, ${ }^{2,7}$ Walter Jaoko, ${ }^{2,7}$ \\ KAVI-ICR Team, ${ }^{2,9}$ and Kelly S. MacDonald ${ }^{1,8,10}$ \\ 1Department of Immunology, University of Toronto, Toronto, Ontario, Canada. ${ }^{2}$ Kenyan AIDS Vaccine Initiative-Institute of Clinical Research (KAVI-ICR), University of Nairobi, Nairobi, Kenya. ${ }^{3}$ Department \\ of Medicine, University of Toronto, Toronto, Ontario, Canada. ${ }^{4}$ University Health Network, Toronto, Ontario, Canada. ${ }^{5}$ Keenan Research Center, St. Michael's Hospital, Toronto, Ontario, Canada. ${ }^{6}$ Community \\ Health Sciences, Max Rady College of Medicine, Rady Faculty of Health Sciences, University of Manitoba, Winnipeg, Manitoba, Canada. ${ }^{7}$ Department of Medical Microbiology, University of Nairobi, Nairobi, \\ Kenya. ${ }^{8}$ Section of Infectious Diseases, Department of Internal Medicine, Max Rady College of Medicine, University of Manitoba, Winnipeg, Manitoba, Canada. ${ }^{9}$ The KAVI-ICR Team is detailed in the \\ Supplemental Acknowledgments. ${ }^{10}$ Department of Medical Microbiology and Infectious Diseases, Max Rady College of Medicine, University of Manitoba, Winnipeg, Manitoba, Canada.
}

BACKGROUND. Varicella-zoster virus (VZV) is under consideration as a promising recombinant viral vector to deliver foreign antigens including HIV. However, new vectors have come under increased scrutiny, since trials with adenovirus serotype 5vectored (Ad5-vectored) HIV vaccine demonstrated increased HIV risk in individuals with pre-immunity to the vector that was thought to be associated with mucosal immune activation (IA). Therefore, given the prospect of developing an HIV/VZV chimeric vaccine, it is particularly important to define the impact of VZV vaccination on IA.

\begin{abstract}
METHODS. Healthy VZV-seropositive Kenyan women $(n=44)$ were immunized with high-dose live attenuated VZV vaccine, and we assessed the expression on CD4 ${ }^{+} \mathrm{T}$ cells isolated from blood, cervix, and rectum of IA markers including CD38 and HLA-DR and of markers of cell migration and tissue retention, as well as the concentration of genital and intestinal cytokines. A delayed-start group $(n=22)$ was used to control for natural variations in these parameters.

RESULTS. Although immunogenic, VZV vaccination did not result in significant difference in the frequency of cervical activated (HLA-DR+CD38+) CD4+ $T$ cells (median $1.61 \%$, IQR $0.93 \%-2.76 \%$ ) at 12 weeks after vaccination when compared with baseline (median $1.58 \%$, IQR $0.75 \%-3.04 \%$ ), the primary outcome for this study. VZV vaccination also had no measurable effect on any of the IA parameters at 4,8 , and 12 weeks after vaccination.
\end{abstract}

CONCLUSION. This study provides the first evidence to our knowledge about the effects of VZV vaccination on human mucosal IA status and supports further evaluation of VZV as a potential vector for an HIV vaccine.

TRIAL REGISTRATION. ClinicalTrials.gov NCT02514018.

FUNDING. Primary support from the Canadian Institutes for Health Research (CIHR). For other sources, see Acknowledgments.

\section{Introduction}

Previous phase IIb/III HIV vaccine trials attest to the challenges faced in human immunodeficiency virus (HIV) vaccine development. The use of adenovirus serotype 5 (Ad5) as a recombinant viral vector to deliver HIV genes not only failed to prevent HIV infection and to reduce viral load following infection in vaccinated individuals (1-3), but also showed a trend toward increased rates of HIV infection in the vaccine arm compared with placebo $(1,2)$. Post hoc analysis showed that the Ad5-based HIV vaccine increased suscep-

Conflict of interest: SMM has received grants for unrelated studies from Merck,

GlaxoSmithKline, Sanofi Pasteur, Pfizer, and Roche-Assurex. SMM has received fees as an advisory board member for Sanofi Pasteur.

License: Copyright 2019, American Society for Clinical Investigation.

Submitted: August 27, 2018; Accepted: November 27, 2018.

Reference information: J Clin Invest. 2019;129(2):875-886.

https://doi.org/10.1172/JCI124473. tibility to HIV infection, especially among those individuals with preexisting antibody immunity to the Ad5 vector $(4,5)$. Although the immune characteristics associated with risk for HIV infection per se have not been completely elucidated, it is known that $\mathrm{CD} 4^{+} \mathrm{T}$ cell activation and phenotypes contribute to the ability to support productive infection. Studies in rhesus macaques showed that the frequency of activated $\mathrm{CCR}^{+} \mathrm{CD}^{+} \mathrm{T}$ cells in the rectal mucosa prior to challenge with simian immunodeficiency virus (SIV) positively correlated with increased risk of infection and SIV peak viremia in the animals (6). Activated CD4 ${ }^{+} \mathrm{T}$ cells, expressing CD38 and HLA$\mathrm{DR}$, were shown to be more susceptible to infection and to favor viral replication when compared with resting $\mathrm{CD}^{+} \mathrm{T}$ cells (7-9). Additionally, genital inflammation measured by an increased level of proinflammatory cytokines was associated with elevated risk of HIV infection $(10,11)$ and a quiescent state of immune activation (IA) linked to resistance to HIV acquisition in highly exposed HIV-1seronegative (HESN) individuals $(9,12,13)$. Genital inflammation 


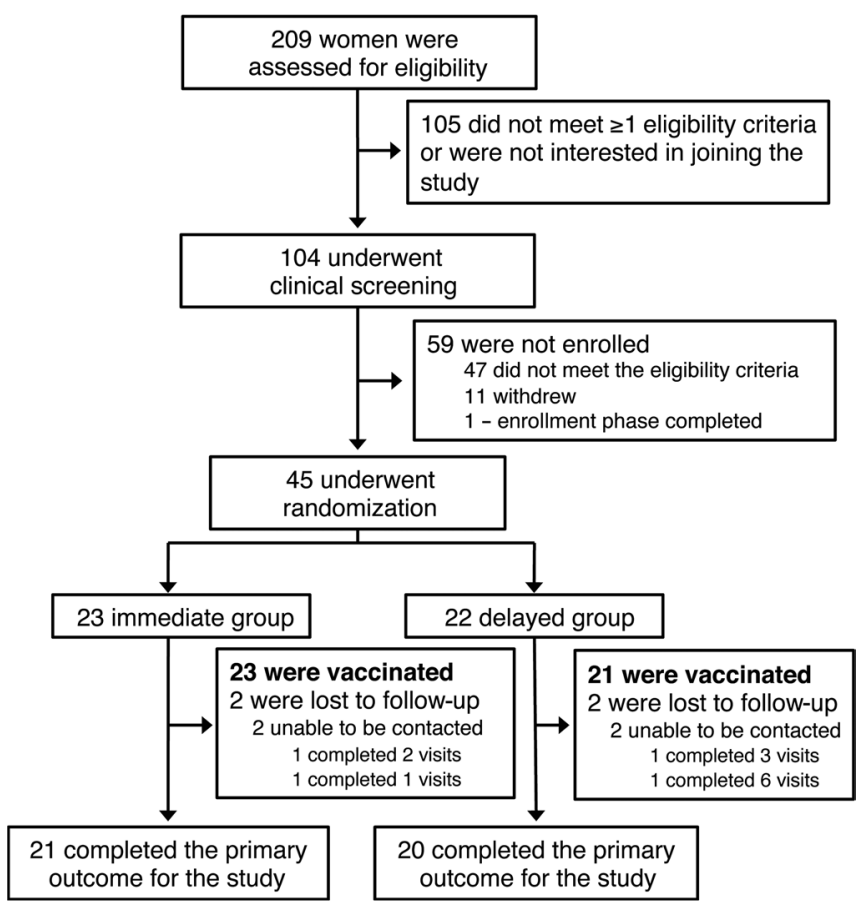

Figure 1. KAVI-VZV 001 enrollment and follow-up diagram.

subverted the protection conferred by the use of $1 \%$ tenofovir gel in the CAPRISA 004 trial (14), and blocking the ability of leukocytes to respond to chemoattractants in the female genital tract (FGT) of humanized mice was shown to prevent systemic dissemination of HIV (15). That the increased rate of HIV acquisition observed in the Ad5-based HIV vaccine trials was associated with increased mucosal IA could not be confirmed, since mucosal specimens were not collected in those studies. Expanded Ad5-specific $\mathrm{CD} 4^{+} \mathrm{T}$ cells from blood expressed increased levels of CCR 5 and the mucosa homing marker $\alpha_{4} \beta_{7}(16,17)$, suggesting that Ad5 vector may promote migration of HIV target cells to the mucosa. A follow-up study in rhesus macaques showed that vaccination with adenovirus vector induced a significant increase in the frequency of activated memory $\mathrm{CD} 4^{+}$ $\mathrm{T}$ cells in rectal mucosa but not in blood (18). There was a reappraisal of the initial Ad5 vaccine rhesus macaque studies $(19,20)$, with a more critical eye. Subsequent nonhuman primate (NHP) studies done with Ad5 demonstrated modest control of viral load (approximately 2 log reduction), with subsequent escape unaccounted for by loss of cytotoxic T lymphocyte control $(21,22)$. Therefore, the limitations of the NHP model and the recognition of the key role mucosal host immune responses against the viral vectors have pushed subsequent vaccines toward much more extensive testing of vectors and of mucosal immunity in humans and macaques.

The use of persistent replicating viral vectors could be instrumental in safely mimicking attenuated HIV antigenic presentation and helping mature and maintain a protective response via vaccine, as suggested by studies in NHP models using CMV as a vector for SIV genes (23-25). However, a detailed assessment of the mucosal immune responses induced by these viral vectors, including IA, is warranted if they are to be used as a chimeric vector bearing HIV antigens $(26,27)$. Additionally, it has been shown that an activated immune microenvironment prior to vaccination can interfere with the efficacy of vaccines (28) and that vaccination may induce sustained $\mathrm{CD} 4^{+} \mathrm{T}$ cell activation, potentially increasing HIV susceptibility in certain populations (29), thus supporting the examination of the IA status in vaccinology.

The long-lived humoral and cellular immunity induced by varicella-zoster virus (VZV) $(30,31)$, its efficacy and safety record as a live attenuated vaccine (31-34) - with vaccination causing viremia in both seronegative and seropositive individuals (35) - combined with its amenability to function as a viral vector expressing foreign antigens $(36,37)$ make VZV a prime candidate to serve as a persistent replicating vector in an HIV vaccine (38). VZV-recombinant vaccine constructs encoding HIV and SIV Env have been previously tested $(39,40)$. Although administration of the VZV-vectored SIV Env vaccine has been linked to accelerated disease progression in rhesus macaques intravenously challenged with SIVsmE660 (40), this result should not be read as a reason to cease the investigation of VZV as a vector in an HIV vaccine. The outcome observed by Staprans et al. is unlikely to be due to VZV-intrinsic features but rather may be due to the study design, which included the following: (i) the use of the highly passaged attenuated human live attenuated $\mathrm{VZV}_{\mathrm{Oka23}}$ vaccine strain in monkeys that was shown not to cross the species barrier, as evidenced by its failure to either induce vaccine-specific $\mathrm{CD} 8^{+} \mathrm{T}$ cell responses or establish latency (so that $\mathrm{VZV}_{\mathrm{Oka23}}$ vaccine acted as a non-replication-competent vaccine); (ii) a single gene product Env in the vaccine that was heterologous to the challenge virus did not induce neutralizing antibodies, and $\mathrm{CD}^{+} \mathrm{T}$ cell proliferation was the sole anti-SIV T cell response induced by the vaccine; (iii) the use of cell-associated instead of cell-free viral vaccine preparation, which may also have increased IA and the availability of activated CD4 ${ }^{+} \mathrm{T}$ cells at the time of challenge. Our group has observed that cynomolgus macaques (CyMs) vaccinated with the VZV-SIV constructs expressing SIV Gag, Pol, and Env and a hybrid of SIV Nef, Tat, and Rev did not exhibit a worse outcome compared with controls; on the contrary, 3 of the 8 vaccine recipients demonstrated complete and sustained SIV viral clearance (undetectable viral load defined as a viral load of fewer than 50 copies in plasma by real-time PCR) after challenge (41). Importantly, VZV-SIV vaccine also did not increase susceptibility to SIV infection in this model. The VZV-vectored constructs tested in the SIV macaque model used as a carrier the less-attenuated parental human strain of $\mathrm{VZV}_{\text {Oka }}$ (Oka-P), which previously was shown able to be able to cross the species barrier and effectively infect CyMs (42).

VZV exposure and subclinical viremia are associated with maintenance of specific cell-mediated immunity able to limit replication of reactivated VZV (43-45), and increased Th1 memory responses induced by VZV vaccination are associated with protection from herpes zoster (46). However, the impact of VZV vaccination with regard to genital and rectal mucosal immunity remains to be defined. We recently showed that VZV-seropositive individuals sustained anti-VZV antibody responses at the genital, oral, and rectal mucosa and also demonstrated that VZV vaccination boosted antiVZV IgG and IgA at the FGT (47). Here, we examined the impact of immunization with the live attenuated zoster vaccine $\left(\mathrm{VZV}_{\mathrm{Oka}}\right.$, Zostavax) on several aspects of IA associated with increased susceptibility to HIV infection longitudinally over a period of 12 weeks, systemically and at both the genital and rectal mucosa, two major 
Table 1. Baseline demographic characteristics, sexual behavior, and HSV-2 serostatus

\begin{tabular}{|c|c|c|c|c|}
\hline Baseline & $\begin{array}{l}\text { Participants enrolled } \\
\qquad(n=45)\end{array}$ & $\begin{array}{l}\text { Immediate group } \\
\quad(n=23)\end{array}$ & $\begin{array}{l}\text { Delayed group } \\
\quad(n=22)\end{array}$ & $P$ \\
\hline \multicolumn{5}{|l|}{ Demographic characteristics } \\
\hline Mean age (SD), yr & $27.0(6.6)$ & $25.6(5.4)$ & $28.5(7.4)$ & 0.212 \\
\hline Median age (IQR), yr & $26(21-30)$ & $26(21-27)$ & $26(23-31)$ & 0.175 \\
\hline \multicolumn{5}{|l|}{ Marital status } \\
\hline Single, no. (\%) & $10(22.2)$ & $5(21.7)$ & $5(22.7)$ & $>0.999$ \\
\hline Married cohabiting, no. (\%) & $33(73.3)$ & $16(69.6)$ & $17(77.3)$ & 0.738 \\
\hline Married living away from partner, no. (\%) & $1(2.2)$ & $1(4.3)$ & 0 & $>0.999$ \\
\hline Divorced or separated, no. (\%) & $1(2.2)$ & $1(4.3)$ & 0 & $>0.999$ \\
\hline \multicolumn{5}{|l|}{ Work status } \\
\hline Unemployed, no. (\%) & $3(6.7)$ & $1(4.3)$ & $2(9.1)$ & 0.608 \\
\hline Housekeeper, no. (\%) & $2(4.4)$ & $1(4.3)$ & $1(4.5)$ & $>0.999$ \\
\hline Casual worker, no. (\%) & $16(35.6)$ & $6(26.1)$ & $10(45.5)$ & 0.221 \\
\hline Professional or technical worker, no. (\%) & $1(2.2)$ & 0 & $1(4.5)$ & 0.489 \\
\hline Self-employed, no. (\%) & $19(42.2)$ & $11(47.8)$ & $8(36.4)$ & 0.550 \\
\hline Student & $4(8.9)$ & $4(17.4)$ & 0 & 0.108 \\
\hline \multicolumn{5}{|l|}{ Education } \\
\hline Completed primary school, no. (\%) & 41 (91.1) & $22(95.7)$ & $19(86.4)$ & 0.346 \\
\hline Completed secondary school, no. (\%) & $16(35.6)$ & $11(47.8)$ & $5(22.7)$ & 0.121 \\
\hline Completed postsecondary school, no. (\%) & $6(13.3)$ & $4(17.4)$ & $2(9.1)$ & 0.665 \\
\hline \multicolumn{5}{|l|}{ Contraception method } \\
\hline Injectable, no. (\%) & $26(57.8)$ & $13(56.5)$ & $13(59.1)$ & $>0.999$ \\
\hline Implant, no. (\%) & $16(35.6)$ & $8(34.8)$ & $8(36.4)$ & $>0.999$ \\
\hline Intrauterine device, no. (\%) & $3(6.7)$ & $2(8.7)$ & $1(4.5)$ & $>0.999$ \\
\hline \multicolumn{5}{|l|}{ Sexual behavior } \\
\hline \multicolumn{5}{|l|}{ Use of condoms with regular partners } \\
\hline Never, no. (\%) & $33(73.3)$ & $18(78.3)$ & $15(68.2)$ & 0.514 \\
\hline Occasionally, no. (\%) & $10(22.2)$ & $5(21.7)$ & $5(22.7)$ & $>0.999$ \\
\hline Frequently, no. (\%) & 0 & 0 & 0 & $>0.999$ \\
\hline Always, no. (\%) & $2(4.4)$ & 0 & $2(9.1)$ & 0.233 \\
\hline \multicolumn{5}{|l|}{ Partner tested positive for HIV } \\
\hline No, no. $(\%)$ & $35(77.8)$ & $18(78.3)$ & $17(77.3)$ & $>0.999$ \\
\hline Do not know, no. (\%) & $10(22.2)$ & $5(21.7)$ & $5(22.7)$ & $>0.999$ \\
\hline \multicolumn{5}{|l|}{ HSV-2 serostatus } \\
\hline HSV-2-positive, no. (\%) & $24(53.3)$ & $8(34.8)$ & $16(72.7)$ & 0.008 \\
\hline
\end{tabular}

HIV coreceptor CCR5, the activation markers CD69 and Ki-67, and markers of cell migration and retention $\left(\alpha_{4} \beta_{7}, \alpha_{4} \beta_{1}\right.$, $\left.\alpha_{\mathrm{E}} \beta_{7}\right)$ in blood, cervix, and rectum. This investigation was conducted in a cohort of healthy Kenyan women at low risk for HIV acquisition and with preexisting immunity to VZV who showed anamnestic VZV-specific humoral responses upon administration of a single dose of $\mathrm{VZV}_{\text {Oka }}$ vaccine (47).

\section{Results}

Participant demographics. Forty-five (45) participants (Figure 1 and Table $1)$ were randomized into immediate $(n=23)$ and delayed $(n=22)$ vaccination groups, as described in Methods and in the published protocol (38) (Figure 2). The inclusion of a delayed group allowed for the adjustment of natural variations in IA parameters independent of vaccination, serving as an internal control group. Blood, cervicovaginal, and rectal specimens were collected longitudinally for the comprehensive examination of several markers of IA before and after vaccination with $\mathrm{VZV}_{\text {Oka }}$ (Figure 2). Vaccine was administered to 44 of 45 (98\%) participants, and 41 of 44 immunized women (93\%) completed the visits required for assessment of the primary study outcome. There were no statistical differences in age, marital status, education level, contraceptive use, or sexual behavior between the two groups (Table 1). While the rate of herpes simplex virus 2 (HSV-2) seropositivity was higher in the delayed than in the immediate group $(P=0.008)$, subsequent adjustment for HSV-2 status did not alter our findings. The median participant age was 26

sites for HIV entry. The primary outcome of the present study was to determine whether 12 weeks after vaccination, the frequency of $\mathrm{CD}^{+} \mathrm{T}$ cells isolated from cervical tissue coexpressing the IA markers CD38 and HLA-DR was significantly changed by vaccination. CD38 ${ }^{+} \mathrm{HLA}_{-} \mathrm{DR}^{+} \mathrm{CD} 4^{+} \mathrm{T}$ cells were previously shown to express high levels of CCR5 and to be highly susceptible to HIV infection (7). Besides, T cells coexpressing these two markers were shown to exhibit high levels of several other markers of IA and lower cytotoxicity (48). Secondary outcomes were to determine changes in a combination of 14 cytokines (including proinflammatory cytokines such as IL-1 $\alpha$, IL-1 $\beta$, and TNF; chemokines IL-8, IP-10, MIP-1 $\alpha$, and MIP$1 \beta$; and the differentiation marker IL-4) measured longitudinally in cervico-vaginal secretion (CVS) and rectal secretion (RS). Tertiary outcomes included the frequency of $\mathrm{CD}^{+} \mathrm{T}$ cells expressing the years (IQR 21-30). The majority of the participants were married (73\%), and 58\% used the progesterone-based injectable hormonal contraceptive depot medroxyprogesterone acetate (DMPA).

No impact of live attenuated $V Z V_{\text {Oka }}$ on genital IA. The frequency of $\mathrm{CD}^{+} \mathrm{T}$ cells expressing the IA markers HLA-DR, CD38, CD69, and Ki-67, as well as the HIV coreceptor CCR5, was measured using flow cytometry (Figure 3A). At 12 weeks after vaccination, there was no significant difference in the frequency of cervical activated (HLA-DR ${ }^{+} \mathrm{CD} 38^{+}$) CD ${ }^{+}$T cells (median 1.61\%, IQR 0.93\%-2.76\%) when compared with week 0 (just prior to vaccination) (median $1.58 \%$, IQR $0.75 \%-3.04 \%$ ) - the primary endpoint for this study (Figure 3B). Based on the SD of the differences in frequency of HLA-DR ${ }^{+} \mathrm{CD}_{3} 8^{+} \mathrm{CD} 4^{+} \mathrm{T}$ cells in the same individual between weeks prior to vaccination (delayed group) $(\mathrm{SD}=3.0 \%, n=59)$, and using 


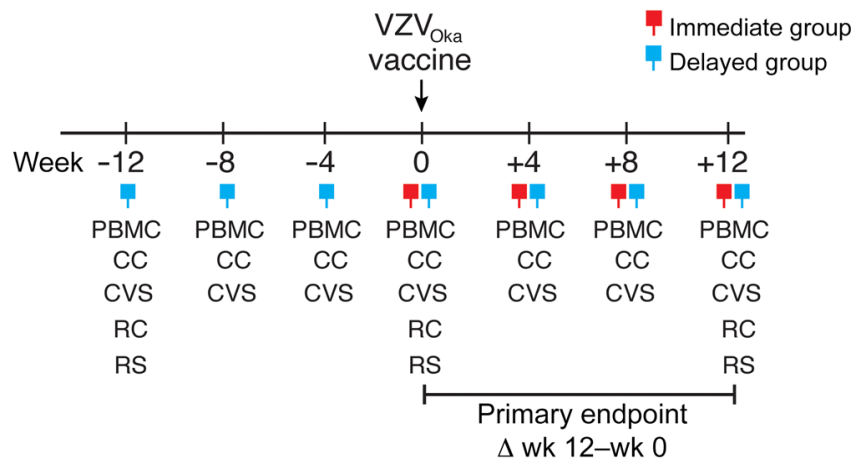

Figure 2. Immunization and sample collection schedule. The primary endpoint was determined between week 12 after vaccination and week 0 . PBMC, peripheral blood mononuclear cells; CC, cervical cells; CVS, cervicovaginal secretion; RC, rectal cells; RS, rectal secretion.

a significance level $(\alpha)$ of 0.05 , we had $80 \%$ power to detect a true difference of at least 1.35 units (frequency [\%] of HLA-DR ${ }^{+} \mathrm{CD} 38^{+}$ $\mathrm{CD}^{+} \mathrm{T}$ cells) between these weeks. $\mathrm{VZV}_{\mathrm{Oka}}$ vaccination also did not lead to changes in the frequency of HLA-DR CD $38^{+} \mathrm{CD} 4^{+} \mathrm{T}$ cells at any of our time points, even prior to adjustment for multiple comparisons (Figure 3B). In addition, no change was seen in the expression of the markers HLA-DR, CD69, and Ki-67, of the HIV coreceptor CCR5 (Figure 3, C-F), or of integrins $\alpha_{4} \beta_{7}, \alpha_{4} \beta_{1}$, and $\alpha_{E} \beta_{7}$ as a result of vaccination (Supplemental Figure 1; supplemental material available online with this article; https://doi.org/10.1172/ JCI124473DS1). The power to detect true changes in the cellular markers assessed in this study is summarized in Supplemental Table 1. Considering the SD of the differences in the frequencies of expression between weeks prior to vaccination (delayed group) in the same individual for each of the markers, the sample size, a significance level $(\alpha)$ of 0.05 , and power $(\beta)$ of $80 \%$, we had the ability to detect a less than 1-fold change (i.e., less than 2 times the baseline value) for all the cellular IA markers expressed on cervical $\mathrm{CD}^{+}{ }^{+} \mathrm{T}$ cells (Supplemental Table 1 ). Therefore, we concluded that VZV vaccination did not cause cervical $\mathrm{CD} 4^{+} \mathrm{T}$ cell IA.

Next the concentration of 14 cytokines in the CVS was assayed. First the correlation between the normalized concentration of these 14 cytokines was assessed, and strong positive correlations (Spearman's correlation $>0.4$ ) between $71 \%$ of the cytokine pairs were identified (Figure $4 \mathrm{~A}$ ). To avoid redundancy and to limit our analysis to a smaller number of covarying components, principal component analysis (PCA) was performed on all cytokines except MIP-1 $\beta$, which was analyzed separately, since it did not correlate with the other cytokines measured in CVS. The first principal component (PC1) explained 63\% of the variability in our dataset (Figure 4B). PC2 and PC3 explained 11\% and 8\% of the variability, respectively (Figure $4 \mathrm{~B}$ ). $\mathrm{VZV}_{\mathrm{Oka}}$ vaccination did not change genital PC1, PC2, or PC3 factor scores (Figure 4,

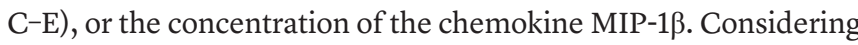
the SD of the differences in the PC factor scores between weeks prior to vaccination (delayed group) in the same individual, the sample size, and $\alpha=0.05$, we were powered to detect fold changes (from week 0) greater than 2.1-, 2.4-, and 1.9-fold for PC1, PC2, and PC3 scores, respectively $(\beta=80 \%)$ (Supplemental Table 2). Longitudinal assessment of the concentration of each FGT cytokine included in the PCA also showed no change after vaccination
(Supplemental Figure 2). Overall, although VZV vaccination significantly increased the concentration of VZV-specific IgG (mIU/ $\mathrm{ml})$ measured in plasma $(P<0.0001)$ and in CVS $(P<0.0001)$ (47), neither T cell flow cytometry data nor FGT cytokine concentrations demonstrated any effect of VZV vaccination on genital IA parameters in healthy VZV-seropositive Kenyan women (Supplemental Figure 3).

Genital IA associated with DMPA use and bacterialvaginosis. Levels of IA prior to vaccination (i.e., in the delayed group) fluctuated over time in some individuals (Figures 3 and 4). The association of these genital IA fluctuations with sexually transmitted infections (STIs), use of DMPA, and presence of bacterial vaginosis (BV) was assessed (Table 2 and Figure 5). Of interest, the use of DMPA as a contraceptive was associated with a reduced PC1 score (TNF- $\alpha$, IL-10, IL-4, IL-1, IL-8, IFN- $\gamma$, IL-6, IL-17A) in the FGT (Figure $5 \mathrm{~A})$. Due to the low incidence of $\mathrm{BV}$ and vulvovaginal candidiasis (VVC) (Table 2), for each participant we grouped all the records during the 12 weeks prior to and after vaccination and examined associations with variability in cytokine and chemokine levels. As shown in Figure 5B, a BV-positive test was associated with significantly elevated PC1 (TNF- $\alpha$, IL-10, IL-4, IL-1, IL-8, IFN- $\gamma$, IL-6, IL-17A) and PC3 (IL-1 $\alpha$, GM-CSF) scores and reduced PC2 (IP-10, MIP-3 $\alpha$, MIP- $1 \alpha$ ) score. A paired analysis using the median PC scores for the BV-negative visits showed similar results, with BV onset associated with significantly lower PC2 score and elevated PC3 score $(P<0.001$, after adjusting for multiple comparisons) (Figure 5C). Women using DMPA as a contraceptive method were 7.7 times less likely to test positive for BV than women using other types of contraceptives $(P=0.019)$. HSV-2 seropositivity was not associated with significant difference for PC1 and PC2 scores, but associated with a significantly elevated median PC3 score, comprising IL-1 $\alpha$ and GM-CSF ( $P=0.027$, after adjusting for multiple comparisons) (Supplemental Figure 4A). VVC was not associated with differences in PC scores (Supplemental Figure 4B).

Live attenuated $V Z V_{O k a}$ did not alter rectal or blood IA status. Given the lack of information regarding the effects of $\mathrm{VZV}_{\mathrm{Oka}}$ vaccination on enteric IA and the importance of the rectal mucosa in HIV transmission, we also examined the impact of $\mathrm{VZV}_{\mathrm{Oka}}$ immunization on markers of IA and on CCR $5, \alpha_{4} \beta_{7}, \alpha_{4} \beta_{1}$, and $\alpha_{\mathrm{E}} \beta_{7}$ expression by rectal $\mathrm{CD} 4^{+} \mathrm{T}$ cells. Rectal biopsy samples were collected at 3 time points in the study: 12 weeks prior to vaccination, on the day of vaccination, and 12 weeks after vaccination. Freshly isolated cells were analyzed by flow cytometry (Figure $6 \mathrm{~A})$. As with cervical CD4 ${ }^{+} \mathrm{T}$ cells, there was no significant elevation of IA in rectal CD $4^{+} \mathrm{T}$ cells due to $\mathrm{VZV}_{\mathrm{Oka}}$ immunization at week 12 after vaccination when compared with the day of vaccination (baseline) (Figure 6, B-E); the frequency of CCR5 ${ }^{+} \mathrm{CD} 4^{+}$ T cells (Figure 6F) and of CD $4^{+} \mathrm{T}$ cells expressing $\alpha_{4}{ }^{+} \beta_{7}{ }^{\text {hi }}, \alpha_{4}{ }^{+} \beta_{1}^{+}$, and $\alpha_{\mathrm{E}}{ }^{+} \beta_{7}{ }^{\text {hi }}$ integrins was also unaltered (Supplemental Figure 5). In the delayed group, a slight decrease in the median frequency of

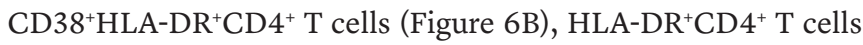
(Figure 6C), and $\alpha_{4}+\beta_{7}{ }^{\text {hi }} \mathrm{CD} 4^{+} \mathrm{T}$ cells (Supplemental Figure $5 \mathrm{~B}$ ) was observed in rectal tissue over the 12 weeks of pre-vaccination follow-up $(P=0.04)$, suggesting that enrollment in the study may potentially be associated with lowering of rectal IA. However, none of these changes remained significant after adjusting for multiple comparisons. 
A

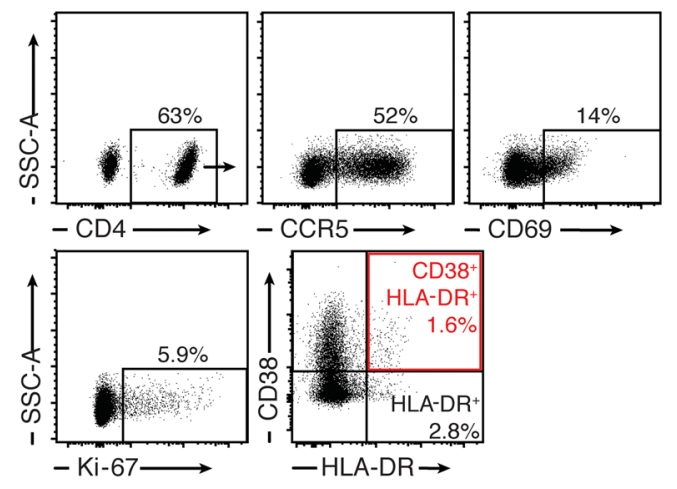

C $\mathrm{HLA}-\mathrm{DR} \mathrm{R}^{+} \mathrm{CD} 4^{+} \mathrm{T}$ cells

$n=22 \quad 21 \quad 22 \quad 44 \quad 43 \quad 41 \quad 40 \quad n=\begin{array}{llll}21 & 40\end{array}$

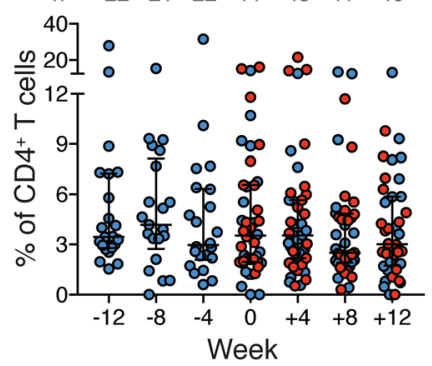

${ }_{201}^{40} 1$ ol o

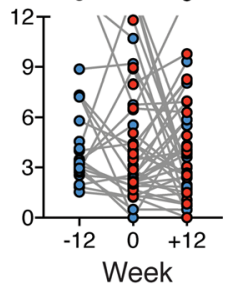

E $\mathrm{Ki}-67^{+} \mathrm{CD} 4^{+} \mathrm{T}$ cells
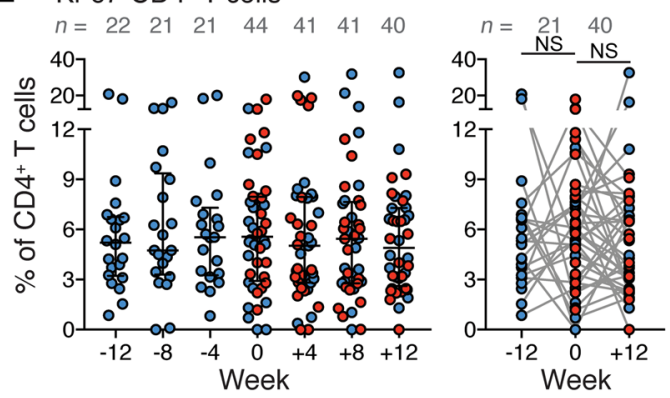

B

- Immediate group oDelayed group

CD38+HLA-DR ${ }^{+}$CD4 ${ }^{+}$T cells
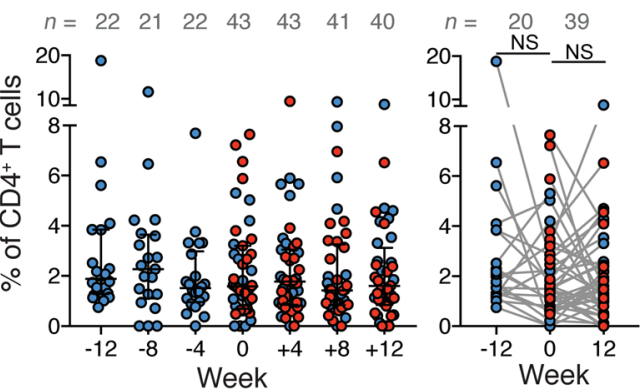

D $\mathrm{CD}_{69}{ }^{+\mathrm{CD}} 4^{+} \mathrm{T}$ cells

$n=\begin{array}{lllllllll}22 & 21 & 22 & 43 & 43 & 41 & 40 & n=20 & 39\end{array}$

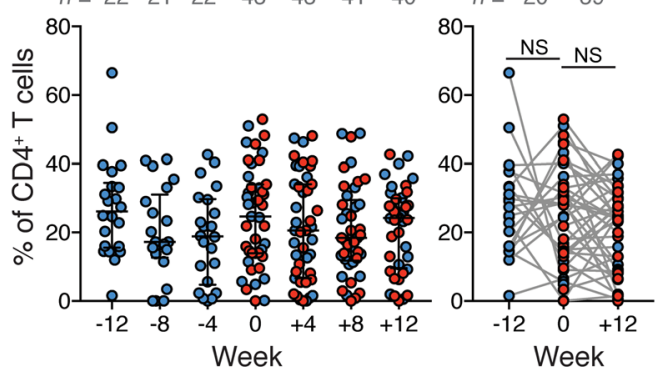

F $\mathrm{CCR} 5{ }^{+} \mathrm{CD} 4^{+} \mathrm{T}$ cells
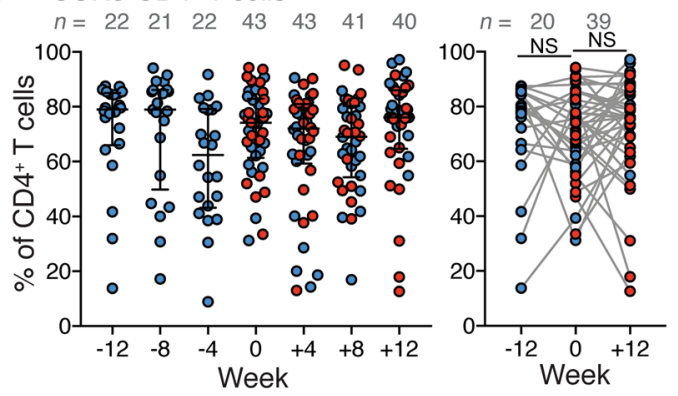

Figure 3. Cervical cellular IA markers. (A) Representative flow cytometry plots for the identification of cervical CD4+ ${ }^{+}$cells, and CCR5 ${ }^{+}$, CD69+, Ki-67+, CD38+HLA-DR ${ }^{+}$, and HLA-DR+ CD4+ T cells. Cells were pre-gated on lymphocytes, singlets, viability by LIVE/DEAD staining, and CD3 ${ }^{+}$cells. Expression of (B) CD38/HLA-DR, (C) HLA-DR, (D) CD69, (E) Ki-67, and (F) CCR5 on cervical CD4 ${ }^{+}$T cells prior to vaccination (weeks -12 to 0 ) and after vaccination (weeks +4 to +12). Graphs to the left show median with IQRs, and graphs to the right the comparison between weeks -12 and 0 and between weeks 0 and +12 for each of the graphs. Individuals in the immediate and delayed groups are shown in red and blue, respectively, and were grouped according to time from vaccination for this analysis. Time points were compared with week 0 using Wilcoxon's signed-rank test. No significant change was observed between any of the time points (unadjusted for multiple comparisons).

We next characterized any changes in the same 14 cytokines in RSs using an approach similar to that used for the FGT (above). We first assessed the correlations between the normalized concentration of these 14 cytokines measured in RS and found strong positive correlations (Spearman's correlation $>0.4$ ) between $86 \%$ of the cytokine pairs (Figure $7 \mathrm{~A}$ ). We next performed PCA on the panel of 14 cytokines. PC1 explained $58 \%$ of the variability in our dataset, whereas PC2 and PC3 explained $14 \%$ and $7 \%$ of the variability, respectively (Figure 7B). The pattern matrices for the PCA in CVS and in RS were similar. The variables encompassing rectal PC1 and PC3 coincided with components of cervico-vaginal PC1 and PC3. However, MIP-1 $\beta$, which was not included in cervico-vaginal PCA, and IL-17A, which was part of cervico-vaginal PC1, were integrated into rectal PC2 (Figure 7B). As in the FGT, $\mathrm{VZV}_{\text {Oka }}$ vaccination did not result in any significant change in the PC1, PC2, or PC3 factor scores at the rectal mucosa (Figure 7, C-E). The study power to detect a true difference in the expression of the cellular markers and in the PC factor scores for the rectal site is shown in Supplemental Tables 1 and 2, respectively. The longitudinal assessment of the concentration of each cytokine included in the PCA of the RSs agreed with these findings by showing no significant change after vaccination (Supplemental Figure 5). At an individual cytokine level, the median rectal concentration of MIP- $1 \alpha$ and MIP- $3 \alpha$ fell 12 weeks after enrollment in the study ( $P=0.044$ and $P=0.005$, respectively), and the median concentration of IL-4 slightly increased ( $P=0.015)$ (Supplemental Figure 6), but these differences were not significant after adjusting for multiple comparisons. Contrary to what was observed for the FGT, the rectal PC scores were independent of use of DMPA as a contraceptive (Supplemental Figure 7). Overall, we conclude that VZV vaccination did not increase rectal IA. 
A

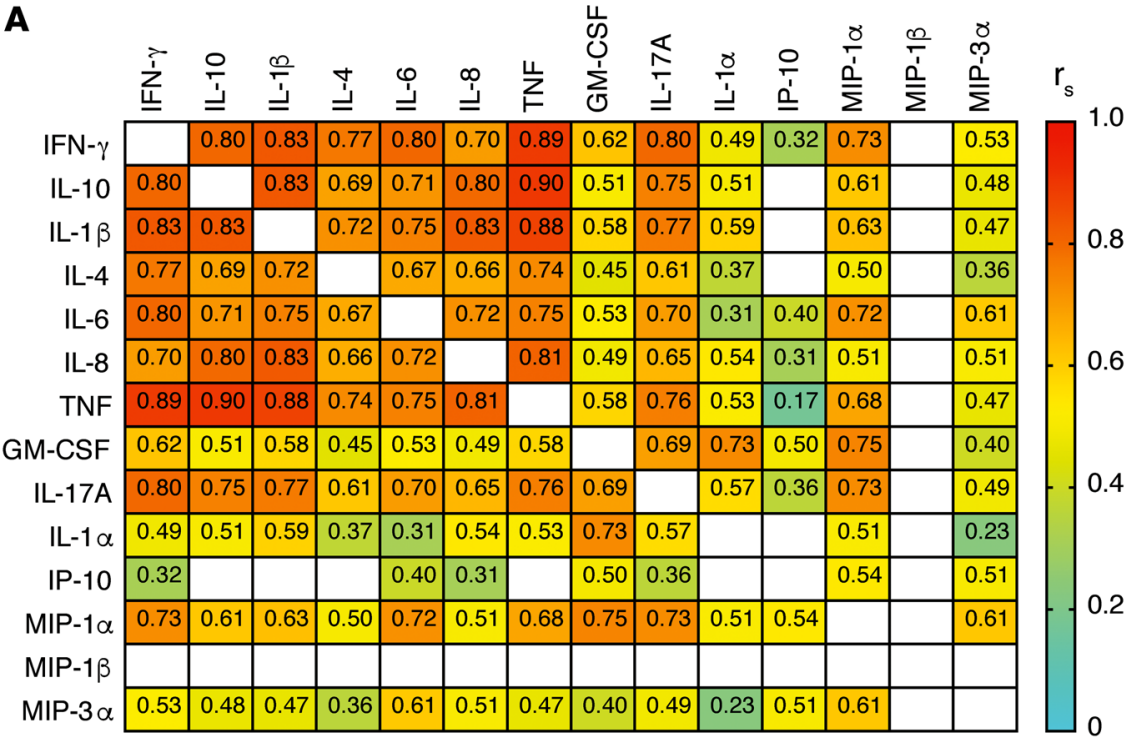

B

\begin{tabular}{|c|c|c|c|c|}
\hline \multicolumn{2}{|c|}{ Pattern matrix } & & \multirow[b]{2}{*}{$\mathrm{PC2}$} & \multirow{3}{*}{$\begin{array}{l}\text { PC3 } \\
8 \%\end{array}$} \\
\hline & & & & \\
\hline & of variance & $63 \%$ & $11 \%$ & \\
\hline \multirow{13}{*}{ 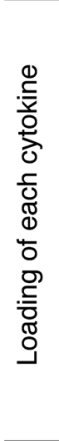 } & TNF & 0.930 & -0.075 & 0.095 \\
\hline & IL-10 & 0.919 & -0.079 & 0.054 \\
\hline & IL-4 & 0.895 & -0.086 & -0.069 \\
\hline & IL-1 $\beta$ & 0.893 & -0.101 & 0.152 \\
\hline & IL-8 & 0.843 & 0.012 & 0.038 \\
\hline & IFN- $\gamma$ & 0.837 & 0.113 & 0.085 \\
\hline & IL-6 & 0.810 & 0.311 & -0.139 \\
\hline & IL-17A & 0.590 & 0.178 & 0.326 \\
\hline & IP-10 & -0.163 & 0.936 & 0.113 \\
\hline & MIP-3 $3 \alpha$ & 0.404 & 0.654 & -0.164 \\
\hline & MIP-1 $1 \alpha$ & 0.336 & 0.510 & 0.351 \\
\hline & IL-1 $\alpha$ & 0.126 & -0.139 & 0.902 \\
\hline & GM-CSF & 0.072 & 0.339 & 0.773 \\
\hline
\end{tabular}

C

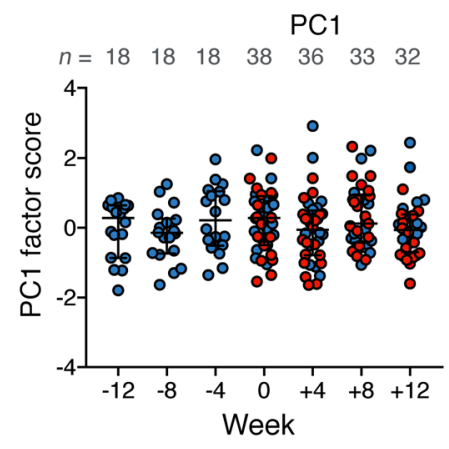

E

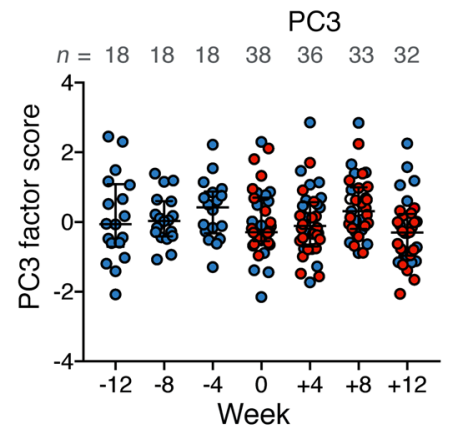

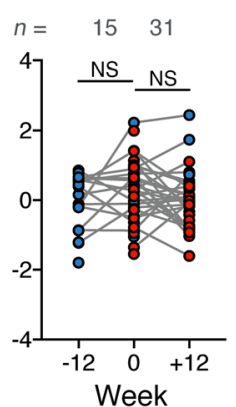

Week
D
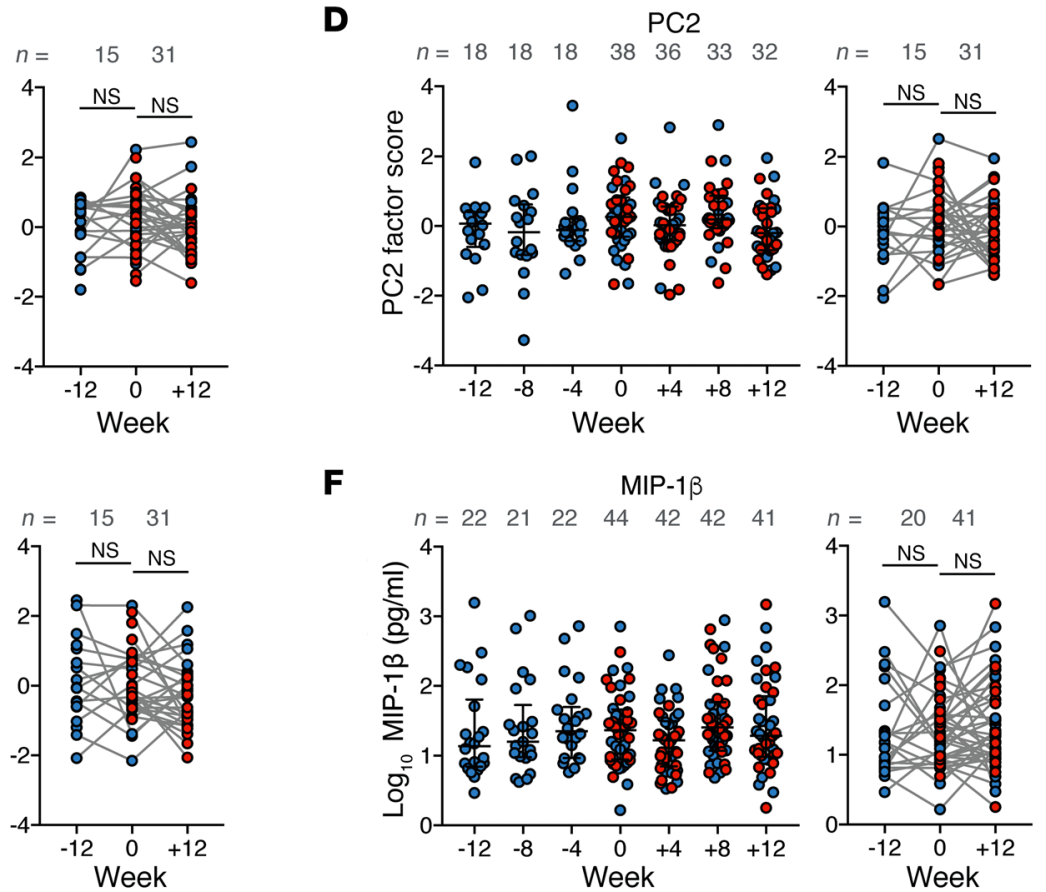

$\mathbf{F}$

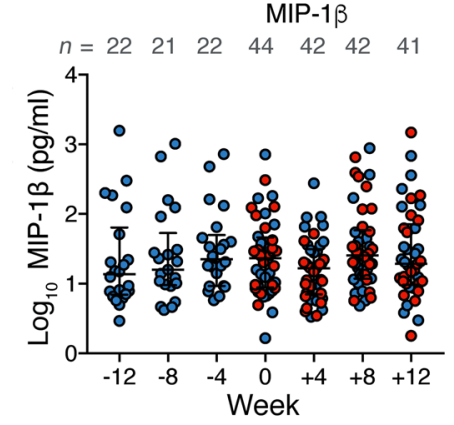

- Immediate group - Delayed group

Figure 4. Cervical soluble IA markers. (A) Spearman's correlation between cervico-vaginal cytokines (using $\log _{10}$-normalized concentrations). Only $r_{s}$ with $P<0.0005$ are shown $(n=234)$. (B) Pattern matrix for the PCA showing loading of each cytokine on the principal components. PC1 explains $63 \%$ of variance, and PC2 and PC3 explain $11 \%$ and $8 \%$ of variance, respectively. Loads greater than 0.450 are highlighted. (C) PC1, (D) PC2, and (E) PC3 factor scores and (F) MIP-1 $\beta$ level prior to vaccination (weeks -12 to 0 ) and after vaccination (weeks +4 to +12 ). Graphs to the left show median with IQR, and graphs to the right the comparison between weeks -12 and 0 and between weeks 0 and +12 for each of the graphs. Individuals in the immediate and delayed groups are shown in red and blue, respectively, and were grouped according to time from vaccination for this analysis. Time points were compared to week 0 using Wilcoxon's signed-rank test. No significant change was observed between any of the time points (unadjusted for multiple comparisons).

A comprehensive assessment of activation and expression of CCR5 and integrins in $\mathrm{CD} 4^{+} \mathrm{T}$ cells isolated from blood also confirmed the absence of deleterious effects related to $\mathrm{VZV}_{\mathrm{Oka}}$ vaccination (Supplemental Figure 8).

\section{Discussion}

Worldwide, HIV transmission predominantly occurs at the mucosal interface and mainly through heterosexual transmission. Mucosal activated $\mathrm{CD} 4^{+} \mathrm{T}$ cells are pivotal for the establishment and dissemination of HIV infection (7, 15, 49-53). Enhanced mucosal IA upon vaccination with Ad5 vector remains the main explanation for the increased rate of HIV infection among vaccine recipients who showed pre-immunity to the vector in the Ad5-vectored HIV vaccine clinical trials, highlighting the importance of examining tissue-specific effects induced by viral vectors $(6,16-18)$. In this light, we comprehensively assessed the impact of $\mathrm{VZV}_{\mathrm{Oka}}$ vaccination on the IA status of both cervico-vaginal and rectal mucosa of Kenyan women with pre-immunity to VZV in 


\section{Table 2. Number of participants who came to the clinic at each time point and respective number of DMPA users; those with BV, VVC, and pelvic inflammatory disease (PID); and HSV-2 positive cases}

\begin{tabular}{|c|c|c|c|c|c|c|c|c|}
\hline Study week & -12 & -8 & -4 & 0 & +4 & +8 & +12 & $\begin{array}{l}\text { Total no. } \\
\text { of records }\end{array}$ \\
\hline Participants, no. & 22 & 21 & 22 & 44 & 42 & 42 & 41 & 234 \\
\hline DMPA, no. & 13 & 13 & 12 & 25 & 25 & 25 & 22 & 135 \\
\hline BV, no. & 2 & 2 & 0 & 2 & 3 & 5 & 3 & 17 \\
\hline VVC, no. & 2 & 1 & 3 & 1 & 3 & 2 & 2 & 14 \\
\hline PID', no. & 0 & 0 & 1 & 0 & 0 & 1 & 0 & 2 \\
\hline HSV- $2^{\mathrm{B}}$, no. & 16 & 15 & 16 & 24 & 24 & 24 & 24 & 143 \\
\hline
\end{tabular}

BV: Nugent score $\geq 7$. PID, pelvic inflammatory disease. ${ }^{A}$ Based on clinical symptoms. ${ }^{B}$ Based on the presence of anti-HSV-2 antibody at screening.

preparation for its further investigation as a vector in an HIV vaccine. As shown here, we did not observe any significant change in the frequency of activated $\mathrm{CD} 4^{+} \mathrm{T}$ cells or in $\mathrm{CCR} 5^{+} \mathrm{CD} 4^{+}$ $\mathrm{T}$ cells at 12 weeks after vaccination at any of the sites. Analysis performed in both circulating and cervical CD $4^{+} \mathrm{T}$ cells as early as 4 weeks after vaccination also did not show significant changes in IA when compared with matched baseline samples collected immediately prior to vaccination (week 0). It is important to highlight that we were powered to detect increases or decreases equal to or greater than the ones shown in Supplemental Table 1, and it is possible that by using a larger sample size, one may detect smaller changes that were imperceptible here.

Enhanced expression of $\alpha_{4} \beta_{7}$ integrin, a mucosal homing marker, has also been shown to contribute to susceptibility to HIV infection and to worsen HIV clinical prognosis (54). Further supporting a role for the integrin, $\alpha_{4} \beta_{7}$ blockade with a monoclonal antibody was recently shown to reduce HIV susceptibility and suppress viral load in NHPs $(55,56)$. Anti- $\alpha_{4} \beta_{7}$ monoclonal antibody therapy, however, was unable to prevent gut $\mathrm{CD} 4^{+} \mathrm{T}$ cell depletion during acute SIV infection in NHPs, with benefits to $\mathrm{CD} 4^{+} \mathrm{T}$ cell reconstitution in gut tissues demonstrated only at later stages of infection (57). Ad5-specific blood CD4 ${ }^{+} \mathrm{T}$ cells exhibited high expression of $\alpha_{4} \beta_{7}$, suggesting that Ad5 vector may promote migration of HIV target cells to the mucosa, increasing their availability at the site of HIV infection. Our results revealed that $\mathrm{VZV}_{\mathrm{Oka}}$ vaccination did not result in an elevated frequency of CD $4^{+} \mathrm{T}$ cells expressing $\alpha_{4} \beta_{7}$ in blood or cervical or rectal mucosa at any time points after vaccination. We have previously shown in this cohort that expression of $\alpha_{\mathrm{E}} \beta_{7}$ may be especially important for $\mathrm{T}$ cell distribution in the rectal mucosa and that $\alpha_{\mathrm{E}}{ }^{+} \beta_{7}{ }^{\text {hi }} \mathrm{CD} 4^{+} \mathrm{T}$ cells express high levels of CCR5 and CD69 (58). Analysis of the frequency of $\alpha_{\mathrm{E}}{ }^{+} \beta_{7}{ }^{\text {hi }} \mathrm{CD} 4^{+}$ $\mathrm{T}$ cells prior to and after vaccination showed that $\mathrm{VZV}_{\mathrm{Oka}}$ vaccination was not associated with any significant change in this cell population in any of the tissues studied here. Additionally, cervical $\mathrm{CD}^{+}$ T cells expressing the integrin $\alpha_{4} \beta_{1}$, previously shown to be readily infected by HIV R5 pseudovirus in an in vitro assay (59), also were not altered by $\mathrm{VZV}_{\text {Oka }}$ vaccination.

Besides cellular markers of IA, genital inflammation measured by an increased level of proinflammatory cytokines is associated with elevated risk of HIV infection $(10,11)$. In agreement with our cellular results, examination of a panel of cytokines - including TNF, IL-1 $\alpha$, IL-1 $\beta$, IL-6, IL-8, IP-10, and MIP-1 $\alpha-$ revealed no significant change in concentration associated with $\mathrm{VZV}_{\mathrm{Oka}}$ vaccination in either CVS or RS. Importantly, all participants remained HIV-1 and HIV-2 negative at the end of our study.

This study also demonstrates that mucosal IA status is highly dynamic and undergoes regular fluctuations, as was evidenced in the 12-week run-in period prior to the administration of the $\mathrm{VZV}_{\mathrm{Oka}}$ vaccine in the delayed group; this reflects the importance of conducting longitudinal studies in relevant human populations with appropriate controls such as a delayed arm. This variability between individuals and even within visits in the same individual is currently being further analyzed. The study will characterize changes to the female genital microbiome over an extended period of 48 weeks and analyze whether they correlate with host changes in mucosal cellular and soluble IA parameters, as well as demographic, contraception, and behavioral factors. Previous studies have shown that the cervico-vaginal microbiota can impact genital inflammation and can increase HIV risk by recruiting HIV
A

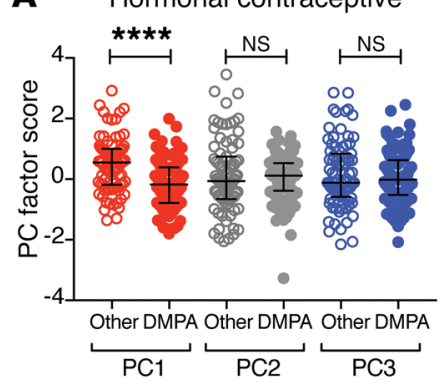

B Bacterial vaginosis - overall

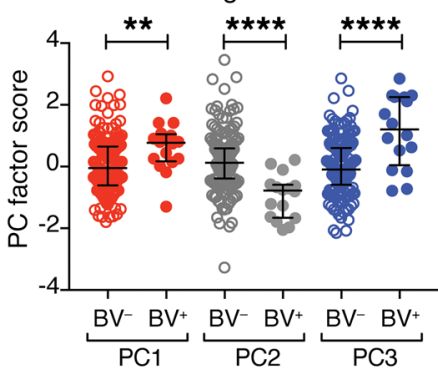

C Bacterial vaginosis - paired

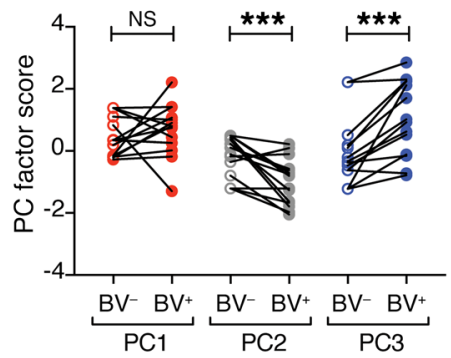

Figure 5. Impact of DMPA use and BV incidence on genital IA. (A) PC factor score comparisons between records for DMPA users ( $n=135)$ and for women using other hormonal contraceptives $(n=99)$. (B) Comparison between PC factor scores for BV+ records $(n=17)$ and BV- records $(n=217)$. Graphs show median with IQRs. ${ }^{* *} P<0.01,{ }^{* * *} P<0.0001$ as determined by a 2-tailed Mann-Whitney $U$ test. (C) Paired comparison between PC factors for BV+ visits and the median for BV- visits for each participant who had BV during this period of the study. ${ }^{* *} P<0.001$ calculated by Friedman's test, followed by Wilcoxon's signed-rank test, and adjusted for multiple comparisons using step-down procedure. 
A

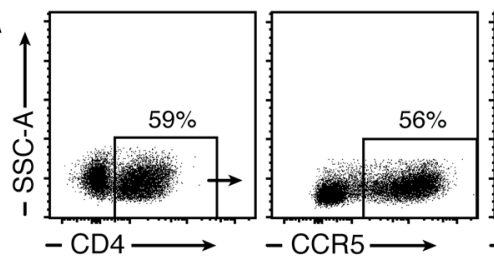

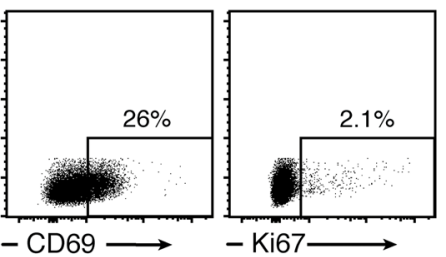

C $\mathrm{HLA}-\mathrm{DR} \mathrm{R}^{+} \mathrm{CD} 4^{+} \mathrm{T}$ cells

B $\mathrm{CD} 38^{+} \mathrm{HLA}-\mathrm{DR}{ }^{+} \mathrm{CD} 4^{+} \mathrm{T}$ cells $n=22 \quad 43 \quad 39 \quad n=20 \quad 37$

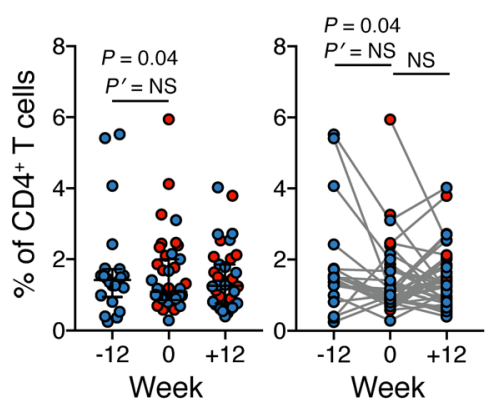

E Ki-67+CD4+ $\mathrm{T}$ cells $n=22 \quad 44 \quad 39 \quad n=21 \quad 38$

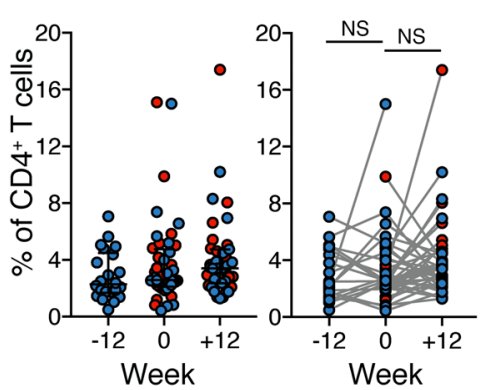

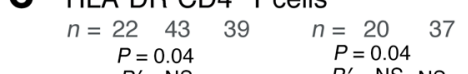

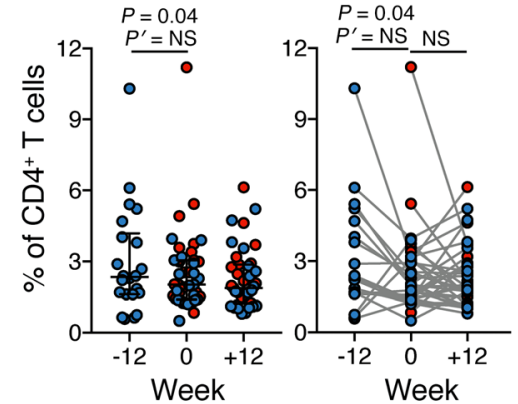

Week

F $\mathrm{CCR} 5{ }^{+} \mathrm{CD} 4^{+} \mathrm{T}$ cells

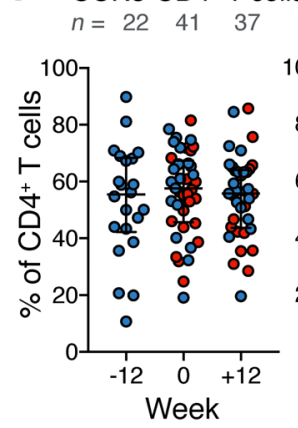

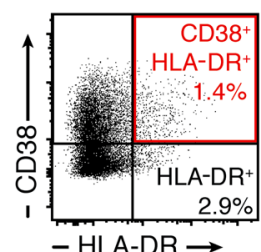

D $\mathrm{CD}^{2} 9^{+} \mathrm{CD} 4^{+} \mathrm{T}$ cells $n=22 \quad 44 \quad 39 \quad n=21 \quad 38$

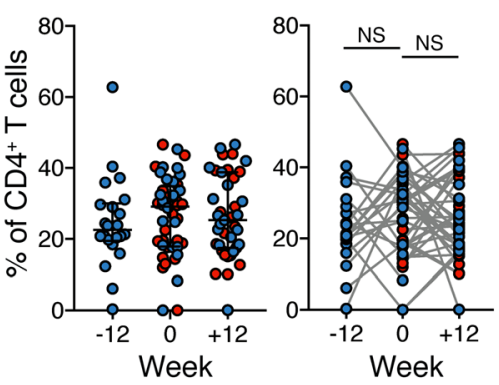

- Immediate group - Delayed group

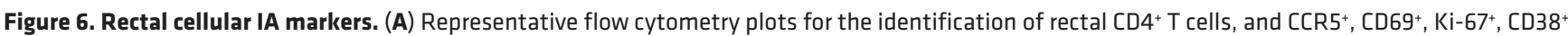
HLA-DR', and HLA-DR+ CD4+T cells. Cells were pre-gated on lymphocytes, singlets, live, and CD3 ${ }^{+}$cells. Expression of (B) CD38'HLA-DR, (C) HLA-DR, (D) CD69, (E) Ki-67, and (F) CCR5 on rectal CD4+ $T$ cells prior to vaccination (weeks -12 and 0 ) and after vaccination (week +12 ). Graphs to the left show median with IQRs, and graphs to the right the comparison between weeks -12 and 0 and between weeks 0 and +12 for each of the graphs. Individuals in the immediate and delayed groups are shown in red and blue, respectively, and were grouped according to time from vaccination for this analysis. Time points were compared with week 0 using Friedman's test, followed by Wilcoxon's signed-rank test. $P$ and $P^{\prime}$ show $P$ values unadjusted and adjusted for multiple comparisons using step-down procedure, respectively.

target cells to the mucosa $(60,61)$. BV and STIs, such as HSV-2 and chlamydia, are associated with elevated genital inflammation and increased risk of HIV infection (62-64). Recent findings by McKinnon et al. demonstrated that genital inflammation can attenuate the protection conferred by $1 \%$ tenofovir gel (14), indicating that the consideration of genital inflammation is also of primary importance when evaluating HIV vaccines.

Participants in this study were drawn from an HIV-uninfected Kenyan population with low rates of partner change, no known commercial sex work, and low rates of STI. These factors may well assist in deciphering the interaction of factors such as DMPA, $\mathrm{BV}$, and HSV-2, as most if not all previous studies have been done in cohorts drawn predominantly from women with complex risk behaviors and higher rates of both viral and bacterial genital infections that persist and reactivate, confounding the analysis of IA.

Of interest, the cumulative analysis of PC scores for all $\mathrm{BV}$ cases in the study compared with all BV-negative records (unpaired analysis), as well as the comparison between the median PC scores for BV-negative and BV-positive scores in a paired manner, showed a significant reduction in PC2 score (comprising IP-10, MIP-3 $\alpha$, and MIP-1 $\alpha$ ) and an increase in PC3 score (including IL-1 $\alpha$ and GM-CSF) for BV-positive compared with BV-negative cases. These results are in agreement with previous findings showing that concomitantly increased IL-1 $\alpha$ and decreased IP-10 concentrations in the FGT predicted the presence of an STI or BV with a quite high predictive value (sensitivity $71 \%$, specificity $74 \%$ ) (65). DMPA use was inversely associated with BV incidence in our cohort, with DMPA users at 7.7 times lower risk for testing positive for BV than women using other contraceptives during the study period. Additionally, we observed a less-proinflammatory profile, as evidenced by a lower PC1 score in DMPA users' CVS than in CVS from women using implants or IUDs as contraceptives, in agreement with previous observations showing that injectable hormonal contraceptives were broadly associated with an immunosuppressive FGT innate immune profile (66). With respect to HSV-2 
A

\begin{tabular}{|c|c|c|c|c|c|c|c|c|c|c|c|c|c|c|}
\hline & $\sum_{\underline{\underline{Z}}}^{\mathbf{T}}$ & $\stackrel{\circ}{\stackrel{\circ}{=}}$ & $\stackrel{\frac{m}{5}}{=}$ & $\stackrel{+\underset{I}{=}}{=}$ & $\stackrel{\varphi}{\stackrel{\varphi}{=}}$ & $\stackrel{\infty}{\stackrel{\infty}{\mu}}$ & $\stackrel{U}{Z}$ & $\begin{array}{l}\text { 岁 } \\
\text { U } \\
\sum_{0}^{1}\end{array}$ & $\frac{\sqrt{5}}{\stackrel{5}{=}}$ & $\stackrel{\frac{\sigma}{\prime}}{=}$ & $\frac{\underline{0}}{\underline{\underline{1}}}$ & $\frac{\frac{\gamma}{1}}{\frac{1}{\Sigma}}$ & $\frac{\frac{m}{\Lambda^{\prime}}}{\frac{\bar{L}}{\Sigma}}$ & 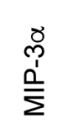 \\
\hline IFN- $\gamma$ & & 0.68 & $\begin{array}{ll}0.61 \\
\end{array}$ & 0.42 & 0.57 & 0.62 & 0.61 & 0.49 & 0.60 & & 0.61 & 0.56 & \begin{tabular}{l|l}
0.59 \\
\end{tabular} & 0.36 \\
\hline IL-10 & 0.68 & & 0.77 & 0.54 & 0.79 & 0.84 & 0.83 & 0.69 & 0.61 & 0.53 & 0.43 & 0.60 & 0.65 & \\
\hline IL-1 $\beta$ & 0.61 & 0.77 & & 0.61 & \begin{tabular}{|l|}
0.78 \\
\end{tabular} & 0.85 & 0.85 & 0.66 & 0.56 & 0.58 & 0.33 & 0.56 & 0.69 & \\
\hline IL-4 & 0.42 & 0.54 & 0.61 & & 0.54 & 0.53 & 0.65 & 0.34 & & 0.35 & & & 0.52 & \\
\hline IL-6 & 0.57 & 0.79 & 0.78 & 0.54 & & 0.85 & 0.83 & 0.53 & 0.49 & 0.47 & 0.37 & 0.55 & 0.57 & \\
\hline IL-8 & 0.62 & 0.84 & 0.85 & 0.53 & 0.85 & & 0.82 & 0.66 & 0.61 & 0.56 & 0.47 & 0.63 & 0.71 & 0.39 \\
\hline TNF & 0.61 & 0.83 & 0.85 & 0.65 & 0.83 & 0.82 & & 0.64 & 0.53 & 0.57 & 0.34 & 0.60 & 0.63 & \\
\hline M-CSF & 0.49 & 0.69 & 0.66 & 0.34 & 0.53 & 0.66 & 0.64 & & 0.54 & 0.77 & 0.34 & 0.64 & 0.57 & \\
\hline IL-17A & 0.60 & 0.61 & 0.56 & & 0.49 & 0.61 & 0.53 & 0.54 & & & 0.64 & 0.61 & 0.63 & 0.55 \\
\hline IL-1 $1 \alpha$ & & 0.53 & 0.58 & 0.35 & 0.47 & 0.56 & 0.57 & 0.77 & & & & 0.46 & 0.34 & \\
\hline IP-10 & 0.61 & 0.43 & 0.33 & & 0.37 & 0.47 & 0.34 & 0.34 & 0.64 & & & 0.65 & 0.68 & 0.72 \\
\hline MIP-1 $\alpha$ & 0.56 & 0.60 & 0.56 & & 0.55 & 0.63 & 0.60 & 0.64 & 0.61 & 0.46 & 0.65 & & 0.69 & 0.56 \\
\hline ИIP-1及 & 0.59 & 0.65 & 0.69 & 0.52 & 0.57 & 0.71 & 0.63 & 0.57 & 0.63 & 0.34 & 0.68 & 0.69 & & 0.56 \\
\hline ИIP-3 $\alpha$ & 0.36 & & & & & 0.39 & & & 0.55 & & 0.72 & 0.56 & 0.56 & \\
\hline
\end{tabular}

B

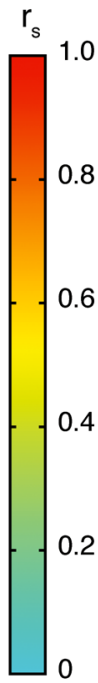

\begin{tabular}{|c|c|c|c|c|}
\hline \multicolumn{2}{|c|}{ Pattern matrix } & & & \\
\hline & & $\overline{\mathrm{PC} 1}$ & PC2 & PC3 \\
\hline \multirow{12}{*}{ 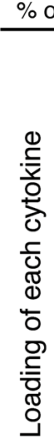 } & variance & $58 \%$ & $14 \%$ & $7 \%$ \\
\hline & $\mathrm{IL}-4$ & 0.972 & -0.142 & -0.202 \\
\hline & TNF & 0.769 & 0.035 & 0.251 \\
\hline & IL-6 & 0.768 & 0.075 & 0.146 \\
\hline & IL-1 $1 \beta$ & 0.706 & 0.098 & 0.268 \\
\hline & IL-10 & 0.632 & 0.173 & 0.294 \\
\hline & IL-8 & 0.615 & 0.240 & 0.285 \\
\hline & IFN- $\gamma$ & 0.504 & 0.450 & -0.008 \\
\hline & IP-10 & 0.035 & 0.944 & -0.125 \\
\hline & MIP-3 $\alpha$ & -0.111 & 0.932 & -0.119 \\
\hline & IL-17A & 0.113 & 0.673 & 0.219 \\
\hline & MIP-1 $\alpha$ & 0.018 & 0.626 & 0.442 \\
\hline & MIP-1 $\beta$ & 0.414 & 0.580 & 0.070 \\
\hline & IL-1 $\alpha$ & 0.089 & -0.207 & 0.934 \\
\hline & GM-CSF & 0.064 & 0.154 & 0.839 \\
\hline
\end{tabular}
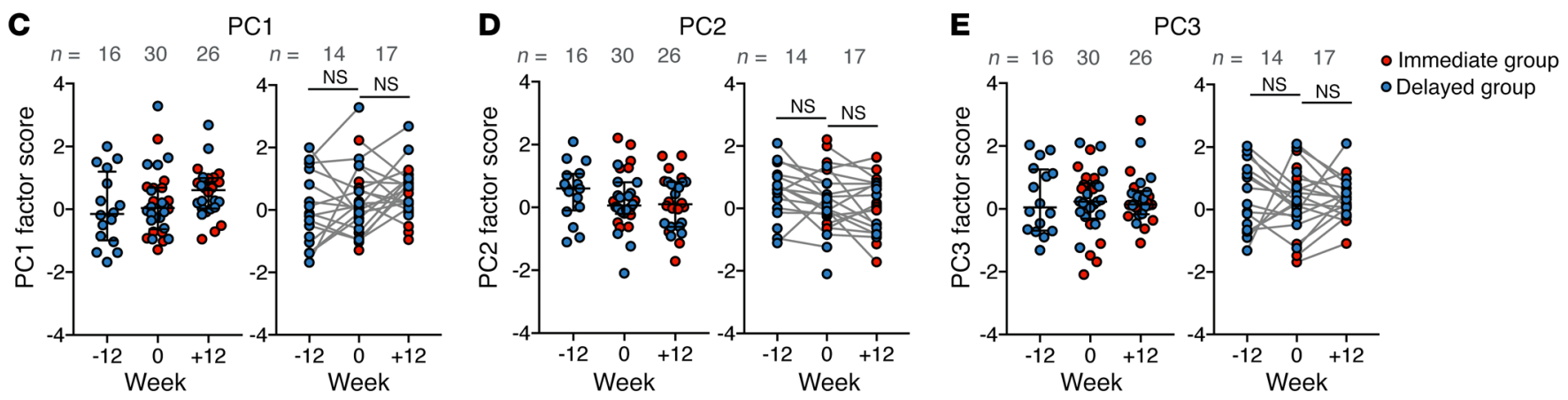

Figure 7. Rectal soluble IA markers. (A) Spearman's correlation between rectal cytokines (using $\log _{10}$-normalized concentrations). Only $r_{s}$ with $P<0.0005$ are shown $(n=107)$. (B) Pattern matrix for the PCA showing loading of each cytokine on the principal components. PC1 explains $58 \%$ of variance, and PC2 and PC3 explain $14 \%$ and $7 \%$ of variance, respectively. Loads greater than 0.450 are highlighted. (C) PC1, (D) PC2, and (E) PC3 factor scores prior to vaccination (weeks -12 and 0 ) and after vaccination (week +12 ). Graphs to the left show median with IQRs, and graphs to the right the comparison between weeks -12 and 0 and between weeks 0 and +12 for each of the graphs. Individuals in the immediate and delayed groups are shown in red and blue, respectively, and were grouped according to time from vaccination for this analysis. Time points were compared with week 0 using Wilcoxon's signed-rank test. No significant change was observed between any of the time points (unadjusted for multiple comparisons).

infection, during the study period we did not observe any clinical indication (such as genital ulcers) of new HSV-2 infections or symptomatic HSV-2 reactivation.

Invasiveness and low acceptance of repeated rectal sampling in clinical trial participants in Kenya precluded more frequent rectal mucosal sampling, which would have added to the ability to compare the cervical IA parameters at multiple time points. A VZV-nonimmune arm would also have been a useful comparator for the study and was contemplated initially; however, screening revealed a very high rate of VZV immunity in this urban population (47), despite the general consensus that VZV seropositivity is lower in adults in the tropics, so recruitment of an adequate sample size of that arm was deemed to be futile. Additionally, since menstrual cycle, hormonal contraceptives, and BV can modulate genital inflammation and HIV susceptibility, future HIV prevention trials should plan to further evaluate the impact of these factors on the protection/risk conferred by the intervention in testing. As administration of live attenuated VZV vaccine is contraindicated for pregnant women, and it is recom- mended that pregnancy be avoided for 3 months following vaccinations, all of our participants were required to use an effective contraceptive method for the duration of the study. Inclusion of a cohort of women not using hormonal contraceptives but consistently using condoms or abstaining from sex would have also added to the study by showing whether hormonal contraceptives can help in reducing/controlling immune activation. However, since recruitment of such a cohort would also imply increasing the sample size and hinder screening, it could not be included in the present trial.

In the effort to develop an HIV vaccine, the incorporation of a recombinant reactivating viral vector would be advantageous compared with other vaccine strategies because it can elicit a longerlasting multifaceted immune response. A pragmatic approach combining modalities and limiting IA is likely to constitute the most effective strategy. VZV is a persistent replicating vector that, like HIV, is able to establish viral latency in the body and possesses several advantageous features for the expression of foreign antigens. Here we provided the first in-depth analysis to our knowledge 
of mucosal IA prior to and following VZV vaccination in a healthy human population. We expanded our findings to cellular and secretory compartments, providing a detailed assessment of mucosal immunity in the FGT and rectal mucosa. We conclude that VZV vaccination was safe and did not increase risk of HIV acquisition, as it did not induce IA either systemically or at the primary portals of entry for HIV. Our findings may pave the way for the use of VZV as a vector for HIV vaccine development initiatives.

\section{Methods}

Study cohort. Participants included healthy, VZV-seropositive adult Kenyan women, not pregnant or breast-feeding, deemed to be at a low risk for HIV infection, who were willing to use an effective contraceptive method for the duration of the study and met other eligibility criteria $(n=45)$; the full study protocol is described elsewhere (38).

Study design. Participants were randomly assigned to either (i) receive the commercially available zoster vaccine (Zostavax, MSD, Merck) immediately after baseline sample collections ("immediate" group) or (ii) be followed for 12 weeks prior to vaccine administration ("delayed" group) (1:1). The randomization was performed sequentially following a statistician-generated randomization list that was securely stored by the clinic manager for the study. After randomization, neither clinic staff nor participants were blinded to assignments. Study samples and data were not identified by randomization status, with the purpose of keeping the laboratory personnel and data analysts blinded to the participants' assignments. The zoster vaccine, containing live attenuated $\mathrm{VZV}_{\text {Oka }}(\geq 19,400 \mathrm{PFU})$, was refrigerated and diluted within 30 minutes of subcutaneous administration as per the manufacturer's directions. Study participants were confirmed as nonpregnant using the QuickVue hCG urine test (Quidel) prior to receiving the vaccine and agreed to use an effective contraceptive method during the study period.

Study outcomes. The primary outcome of the KAVI-VZV 001 trial was cervical mucosal CD $4^{+} \mathrm{T}$ cell IA after $\mathrm{VZV}_{\mathrm{Oka}}$ vaccination, defined as the change in frequency of cervical $\mathrm{CD} 4^{+} \mathrm{T}$ cells coexpressing $\mathrm{CD} 38$ and HLA-DR between weeks 0 and 12 after vaccination compared with the changes observed in the 12 -week period that preceded vaccination in the delayed group. Secondary and tertiary outcomes included cytokine levels in cervico-vaginal and RSs, and other markers of cellular IA (expression of HLA-DR, CD69, Ki-67) in blood, cervical, and rectal $\mathrm{CD}^{+}{ }^{+} \mathrm{T}$ cells before and after vaccination with $\mathrm{VZV}_{\mathrm{Oka}}(38)$.

Sample collection. Sample collection and processing are described in full elsewhere (38). Briefly, blood was collected using BD Vacutainer heparin tubes for the isolation of PBMCs using a standard Ficoll gradient (Histopaque-1077 Hybri-Max, Sigma-Aldrich). The collection of CVS was performed using a flexible plastic cup (Softcup, Instead) inserted into the vagina for 20 minutes. Cervical cells were collected using 2 cytobrushes (Digene, QIAGEN), each inserted into the cervical canal and turned $360^{\circ}$ once. RSs and rectal biopsies were collected using a proctoscope. RSs were collected using 2 sponges (Merocel, Medtronic), and rectal biopsies (10 punches) were collected using a Sarratt disposable forceps (STE1500, Stericom).

Immunophenotyping of cells. PBMCs, cervical cells, and rectal cells were stained immediately with the LIVE/DEAD Far Red Cell Stain Kit (Invitrogen), and viable cells were labeled with the following monoclonal antibodies: CD3 APCeFluor780 (SK7), HLA-DR FITC (L243), CD49d PE (9F10), Ki-67 PECy7 (20Raj1) from eBioscience; CD4 BV510 (SK3), CD38 PE-CF594 (HIT2), CCR5 BV421 (2D7) from BD
Horizon; and CD69 (FN50) and $\beta 7$ PECy5 (FIB504) from BD Pharmingen. All antibodies were titrated before use. Rainbow beads (Spherotech) were used to ensure that the data collection remained consistent throughout the study. Samples were acquired on an LSR II flow cytometer using the DiVa software package (BD Biosciences). Analysis of the acquired data was performed using FlowJo software (Tree Star Inc.).

Measurement of cytokine and chemokine concentrations. Fourteen cytokines and chemokines (IL-1 $\alpha$, IL-1 $\beta$, IL-6, IL-8, IL-10, IL-17A, IFN- $\gamma$, GM-CSF, IP-10, MIP-1 $\alpha$, MIP-1 $\beta$, MIP-3 $\alpha$, TNF- $\alpha$, and IL-4) were measured in all CVS and RS samples using the electrochemiluminescence U-Plex system (Meso Scale Discovery). All samples underwent a single freeze-thaw cycle and were run in duplicate according to the manufacturer's instructions. A quality control was included with every plate to ensure reproducibility. Cytokine measurements below or over the limit of detection for the method were included in the analysis if they were in the fit curve range, and the $R^{2}$ for the curve was $>0.999$.

Genital infection diagnosis. BV and VVC were screened at every visit, using a Gram-stained vaginal smear; BV was diagnosed using Nugent's criteria (67), and VVC as the presence of yeast forms with a vaginal $\mathrm{pH}<4$ (68). Participants were tested for STIs at screening. Neisseria gonorrhoeae and Chlamydia trachomatis were tested using the GeneXpert CT/NG test (Cepheid), and Trichomonas vaginalis was diagnosed using wet prep $\mathrm{pH}>4$ and InPouch TV (BioMed) bioculture system. Syphilis was tested using the rapid plasma reagin (RPR) test and confirmed by Treponema pallidum particle agglutination assay (TPHA; Thermo Fisher Scientific). HIV-1 and HIV-2 testing was performed 3 times during the study using 2 rapid tests, Determine HIV-1/2 (Alere Medical Co.) and Uni-Gold HIV (Trinity Biotech), and Bioelisa $\mathrm{HIV}-1+2 \mathrm{Ag} / \mathrm{Ab}$ (Biokit S.A.) as a confirmatory test. HSV-2 serostatus was determined in plasma samples collected at enrollment using a clinically validated Western blot analysis performed at the National Microbiology Laboratory at the Public Health Agency of Canada.

Statistics. Fisher's exact test was used to compare categorical data among groups. Two-tailed Mann-Whitney $U$ test was used to compare independent samples. Wilcoxon's signed-rank test was used to compare responses between 2 time points. The sample size was calculated based on the SD of frequencies of cervical CD $38^{+} \mathrm{HLA}^{-D R^{+}} \mathrm{CD} 4^{+}$ $\mathrm{T}$ cells measured between subsequent visits from a previous cohort, and aiming to achieve $80 \%$ power to detect changes of 1 SD in frequency, using 2-tailed test with a significance level $(\alpha)$ of 0.05 and assuming a $10 \%$ dropout rate (38). The numeric fold change was calculated as the ratio of the true mean difference able to be detected between week 12 and week 0 relative to the initial value (week 0). For example, an initial value of 2 and a value of 4 at week 12 corresponded to a 1-fold change (or equivalently, a change of 2 times). Spearman's correlation was used to determine the association between variables. Logistic regression was used to estimate the effect of DMPA use on BV incidence. Cytokine expression was evaluated using PCA on the normalized $\left(\log _{10}\right)$ cytokine concentrations, aiming to identify the main components that could explain the data variability while reducing the dimensionality of the cytokine data set. Sampling adequacy for PCA was tested using a correlation matrix for 14 cytokines, KaiserMeyer-Olkin measure, and by Bartletts's test of sphericity. Extraction was performed based on an eigenvalues $>1.0$, ensuring that those did not exceed the eigenvalues generated by our parallel analysis (Monte Carlo PCA) and using oblimin with Kaiser normalization as a rotation method. Factor scores were generated using Bartlett's approach. 
A $P$ value less than 0.05 was considered statistically significant, with ${ }^{*} P<0.05,{ }^{* *} P<0.01,{ }^{* * *} P<0.001$, and ${ }^{* * *} P<0.0001$. Unadjusted and adjusted $P$ values are specified in the figures. Results were adjusted for multiple comparisons using the Holm's step-down procedure. Statistical analyses were performed using IBM SPSS Statistics (v.23). Graphs were generated using Prism 6 (GraphPad) software.

Study approval. This study was reviewed and approved by the Kenyatta National Hospital/University of Nairobi Ethics Research Committee (KNH/UoN ERC), the University of Toronto Research Ethics Board, and by the Kenyan Pharmacy and Poisons Board. KAVIVZV-001 is registered with ClinicalTrials.gov (NCT02514018). All participants included in this study provided written informed consent.

\section{Author contributions}

KSM conceived the study. KSM, CTP, WJ, OA, MAO, and RK designed and coordinated the study. The KAVI-ICR Team, CTP, and BF collected and/or generated the study data. CTP and SMM performed the statistical analysis of the data. CTP, KSM, MAO, and $\mathrm{KR}$ were involved in the analysis or interpretation of the data. All authors contributed to the writing/reviewing of the manuscript.

\section{Acknowledgments}

The authors thank all study participants, as well as David Willer, Sharon Walmsley, Janet Raboud, and Jennifer Gommerman for their scientific advice; Shariq Mujib for proofreading the manuscript; and Christiaan Righolt for statistical advice. We want to thank Alberto Severini for the assessment of HSV-2 status in our cohort, and Fatima Csordas and Jacqueline Chan for laboratory and administrative assistance in Toronto. We also want to thank Marcelo A. Ventura for his artwork used for the graphical abstract. This study was funded by the Canadian Institutes for Health Research (grant THA-11960). CTP was supported by a CIHR Vanier Canada Graduate Scholarship, a Delta Kappa Gamma Society World Fellowship, and an Ontario Graduate Scholarship. KSM was funded by an Ontario HIV Treatment Network (OHTN) Senior Investigator Award. KSM is currently supported by the University of Manitoba Department of Medicine H.E. Sellers Research Chair. MAO was supported by OHTN. SMM is a Canada Research Chair in Pharmacoepidemiology and Vaccine Evaluation. See Supplemental Acknowledgments for KAVI-ICR Team details.

Address correspondence to: Kelly S. MacDonald, Office Room 501, Basic Medical Sciences Building, 745 Bannatyne Avenue, Winnipeg, Manitoba, R3E0J9, Canada. Phone: 204.977.5680; Email: kelly.macdonald@umanitoba.ca. Or to: Catia T. Perciani, Room 6368 Medical Science Building, 1 King's College Circle, Toronto, Ontario, M5S1A8, Canada. Phone: 416.946.0277; Email: c.perciani@mail.utoronto.ca.
1. Buchbinder SP, et al. Efficacy assessment of a cell-mediated immunity HIV-1 vaccine (the Step Study): a double-blind, randomised, placebo-controlled, test-of-concept trial. Lancet. 2008;372(9653):1881-1893.

2. Gray GE, et al. Recombinant adenovirus type $5 \mathrm{HIV}$ gag/pol/nef vaccine in South Africa: unblinded, long-term follow-up of the phase $2 \mathrm{~b}$ HVTN 503/Phambili study. Lancet Infect Dis. 2014;14(5):388-396.

3. Hammer SM, et al. Efficacy trial of a DNA/ rAd5 HIV-1 preventive vaccine. $N$ Engl J Med. 2013;369(22):2083-2092.

4. Duerr A, et al. Extended follow-up confirms early vaccine-enhanced risk of HIV acquisition and demonstrates waning effect over time among participants in a randomized trial of recombinant adenovirus HIV vaccine (Step Study). J Infect Dis. 2012;206(2):258-266.

5. Gray G, Buchbinder S, Duerr A. Overview of STEP and Phambili trial results: two phase IIb test-of-concept studies investigating the efficacy of MRK adenovirus type $5 \mathrm{gag} / \mathrm{pol} / \mathrm{nef}$ subtype B HIV vaccine. Curr Opin HIV AIDS. 2010;5(5):357-361.

6. Carnathan DG, et al. Activated CD4+CCR5+ T cells in the rectum predict increased SIV acquisition in SIVGag/Tat-vaccinated rhesus macaques. Proc Natl Acad Sci U S A. 2015;112(2):518-523.

7. Meditz AL, et al. HLA-DR+ CD38+CD4+ T lymphocytes have elevated CCR5 expression and produce the majority of R5-tropic HIV-1 RNA in vivo. J Virol. 2011;85(19):10189-10200.

8. Stevenson M, Stanwick TL, Dempsey MP, Lamonica CA. HIV-1 replication is controlled at the level of $\mathrm{T}$ cell activation and proviral integration. ЕMBO J.1990;9(5):1551-1560.
9. Bégaud E, et al. Reduced CD4 T cell activation and in vitro susceptibility to HIV-1 infection in exposed uninfected Central Africans. Retrovirology. 2006;3:35.

10. Masson L, et al. Genital inflammation and the risk of HIV acquisition in women. Clin Infect Dis. 2015;61(2):260-269.

11. Lajoie J, et al. A distinct cytokine and chemokine profile at the genital mucosa is associated with HIV-1 protection among HIV-exposed seronegative commercial sex workers. Mucosal Immunol. 2012;5(3):277-287.

12. Tran HK, et al. Systemic immune activation in HIV-1-exposed uninfected Vietnamese intravascular drug users. AIDS Res Hum Retroviruses. 2006;22(3):255-261.

13. Lajoie J, et al. Association of sex work with reduced activation of the mucosal immune system. J Infect Dis. 2014;210(2):319-329.

14. McKinnon LR, et al. Genital inflammation undermines the effectiveness of tenofovir gel in preventing HIV acquisition in women. Nat Med. 2018;24(4):491-496.

15. Deruaz M, et al. Chemoattractant-mediated leukocyte trafficking enables HIV dissemination from the genital mucosa. JCI Insight. 2017;2(7):e88533.

16. $\mathrm{Hu} \mathrm{H}$, et al. Preferential infection of human Ad5-specific CD4 T cells by HIV in Ad5 naturally exposed and recombinant Ad5-HIV vaccinated individuals. Proc Natl Acad Sci US A. 2014;111(37):13439-13444.

17. Auclair S, et al. Distinct susceptibility of HIV vaccine vector-induced CD4 T cells to HIV infection. PLoS Pathog. 2018;14(2):e1006888.

18. Bukh I, et al. Increased mucosal CD4+ T cell activation in rhesus macaques following vac- cination with an adenoviral vector. $J$ Virol. 2014;88(15):8468-8478.

19. Shiver JW, et al. Replication-incompetent adenoviral vaccine vector elicits effective anti-immunodeficiency-virus immunity. Nature. 2002;415(6869):331-335.

20. Liang X, et al. Vectored Gag and Env but not Tat show efficacy against simian-human immunodeficiency virus $89.6 \mathrm{P}$ challenge in Mamu-A*01-negative rhesus monkeys. J Virol. 2005;79(19):12321-12331.

21. Casimiro DR, et al. Efficacy of multivalent adenovirus-based vaccine against simian immunodeficiency virus challenge. J Virol. 2010;84(6):2996-3003.

22. McDermott AB, et al. Cytotoxic T-lymphocyte escape does not always explain the transient control of simian immunodeficiency virus SIVmac239 viremia in adenovirus-boosted and DNA-primed Mamu-A*01-positive rhesus macaques. JVirol. 2005;79(24):15556-15566.

23. Hansen SG, et al. Profound early control of highly pathogenic SIV by an effector memory T-cell vaccine. Nature. 2011;473(7348):523-527.

24. Hansen SG, et al. Immune clearance of highly pathogenic SIV infection. Nature. 2013;502(7469):100-104.

25. Hansen SG, et al. Effector memory T cell responses are associated with protection of rhesus monkeys from mucosal simian immunodeficiency virus challenge. Nat Med. 2009;15(3):293-299.

26. Fauci AS, Marovich MA, Dieffenbach CW, Hunter E, Buchbinder SP. Immunology. Immune activation with HIV vaccines. Science. 2014;344(6179):49-51.

27. D’Souza MP, Yang OO. Adenovirus vectors as HIV-1 vaccines: where are we? What next? AIDS 
2015;29(4):395-400.

28. Muyanja E, et al. Immune activation alters cellular and humoral responses to yellow fever 17D vaccine. J Clin Invest. 2014;124(7):3147-3158.

29. Gasper MA, et al. BCG vaccination induces HIV target cell activation in HIV-exposed infants in a randomized trial. JCI Insight. 2017;2(7):e91963.

30. Arvin AM. Humoral and cellular immunity to varicella-zoster virus: an overview. JInfect Dis. 2008;197 Suppl 2:S58-S60.

31. Levin MJ, et al. Varicella-zoster virus-specific immune responses in elderly recipients of a herpes zoster vaccine. J Infect Dis. 2008;197(6):825-835.

32. Takahashi M. Effectiveness of live varicella vaccine. Expert Opin Biol Ther. 2004;4(2):199-216.

33. Shapiro ED, et al. Effectiveness of 2 doses of varicella vaccine in children. J Infect Dis. 2011;203(3):312-315.

34. Oxman MN, et al. A vaccine to prevent herpes zoster and postherpetic neuralgia in older adults. N Engl JMed. 2005;352(22):2271-2284.

35. Levin MJ, et al. Varicella-zoster virus dna in blood after administration of herpes zoster vaccine. J Infect Dis. 2018;217(7):1055-1059.

36. Matsuura $M$, et al. Novel polyvalent live vaccine against varicella-zoster and mumps virus infections. Microbiol Immunol. 2013;57(10):704-714.

37. Gray WL. Recombinant varicella-zoster virus vaccines as platforms for expression of foreign antigens. Adv Virol. 2013;2013:219439.

38. Perciani CT, et al. Protocol of a randomised controlled trial characterising the immune responses induced by varicella-zoster virus $(\mathrm{VZV})$ vaccination in healthy Kenyan women: setting the stage for a potential VZV-based HIV vaccine. BMJ Open. 2017;7(9):e017391.

39. Shiraki K, et al. Construction of Oka varicella vaccine expressing human immunodeficiency virus env antigen. J Med Virol. 2001;64(2):89-95.

40. Staprans SI, et al. Enhanced SIV replication and accelerated progression to AIDS in macaques primed to mount a CD4 $\mathrm{T}$ cell response to the SIV envelope protein. Proc Natl Acad Sci U S A. 2004;101(35):13026-13031.

41. Perciani CT, et al. Mechanisms of protection observed with varicella zoster virus as a vaccine vector in the siv macaque model. AIDS Res Hum Retroviruses. 2014;30(S1):a5075.

42. Willer DO, et al. Experimental infection of cynomolgus macaques (Macaca fascicularis) with human varicella-zoster virus. J Virol.
2012;86(7):3626-3634.

43. Wilson A, Sharp M, Koropchak CM, Ting SF, Arvin AM. Subclinical varicella-zoster virus viremia, herpes zoster, and T lymphocyte immunity to varicella-zoster viral antigens after bone marrow transplantation. J Infect Dis. 1992;165(1):119-126.

44. Papaevangelou V, et al. Subclinical VZV reactivation in immunocompetent children hospitalized in the ICU associated with prolonged fever duration. Clin Microbiol Infect. 2013;19(5):E245-E251.

45. Cohrs RJ, Gilden DH. Prevalence and abundance of latently transcribed varicella-zoster virus genes in human ganglia. J Virol. 2007;81(6):2950-2956.

46. Levin MJ, et al. Th1 memory differentiates recombinant from live herpes zoster vaccines. J Clin Invest. 2018;128(10):4429-4440.

47. Perciani CT, et al. Live attenuated zoster vaccine boosts varicella zoster virus (VZV)-specific humoral responses systemically and at the cervicovaginal mucosa of kenyan VZV-seropositive women. J Infect Dis. 2018;218(8):1210-1218.

48. Hua S, et al. Potential role for HIV-specific CD38-/HLA-DR+ CD8+ T cells in viral suppression and cytotoxicity in HIV controllers. PLOS One. 2014;9(7):e101920.

49. Li Q, et al. Glycerol monolaurate prevents mucosal SIV transmission. Nature. 2009;458(7241):1034-1038.

50. Hladik F, et al. Initial events in establishing vaginal entry and infection by human immunodeficiency virus type-1. Immunity. 2007;26(2):257-270.

51. Saba E, et al. HIV-1 sexual transmission: early events of HIV-1 infection of human cervico-vaginal tissue in an optimized ex vivo model. Mucosal Immunol. 2010;3(3):280-290.

52. Haase AT. Targeting early infection to prevent HIV-1 mucosal transmission. Nature. 2010;464(7286):217-223.

53. Haase AT. Early events in sexual transmission of HIV and SIV and opportunities for interventions. Annu Rev Med. 2011;62:127-139.

54. Sivro A, et al. Integrin $\alpha 4 \beta 7$ expression on peripheral blood CD4+ T cells predicts HIV acquisition and disease progression outcomes. Sci Transl Med. 2018;10(425):eaam6354.

55. Byrareddy SN, et al. Targeting $\alpha 4 \beta 7$ integrin reduces mucosal transmission of simian immunodeficiency virus and protects gut-associated lymphoid tissue from infection. Nat Med. 2014;20(12):1397-1400.
56. Byrareddy SN, et al. Sustained virologic control in SIV+ macaques after antiretroviral and $\alpha 4 \beta 7$ antibody therapy. Science. 2016;354(6309):197-202.

57. Santangelo PJ, et al. Early treatment of SIV+ macaques with an $\alpha 4 \beta 7 \mathrm{mAb}$ alters virus distribution and preserves CD4+ T cells in later stages of infection. Mucosal Immunol. 2018;11(3):932-946.

58. Perciani CT, et al. $\alpha E \beta 7, \alpha 4 \beta 7$ and $\alpha 4 \beta 1$ integrin contributions to $\mathrm{T}$ cell distribution in blood, cervix and rectal tissues: potential implications for HIV transmission. PLoS One. 2018;13(2):e0192482.

59. Joag VR, et al. Identification of preferential CD4+ T-cell targets for HIV infection in the cervix. Mucosal Immunol. 2016;9(1):1-12.

60. Anahtar MN, et al. Cervicovaginal bacteria are a major modulator of host inflammatory responses in the female genital tract. Immunity. 2015;42(5):965-976.

61. Gosmann C, et al. Lactobacillus-deficient cervicovaginal bacterial communities are associated with increased HIV acquisition in young South African women. Immunity. 2017;46(1):29-37.

62. Wald A, Link K. Risk of human immunodeficiency virus infection in herpes simplex virus type 2-seropositive persons: a meta-analysis. J Infect Dis. 2002;185(1):45-52.

63. Van de Perre P, et al. Herpes simplex virus and HIV-1: deciphering viral synergy. Lancet Infect Dis. 2008;8(8):490-497.

64. Masson L, et al. Defining genital tract cytokine signatures of sexually transmitted infections and bacterial vaginosis in women at high risk of HIV infection: a cross-sectional study. Sex Transm Infect. 2014;90(8):580-587.

65. Masson L, et al. Inflammatory cytokine biomarkers to identify women with asymptomatic sexually transmitted infections and bacterial vaginosis who are at high risk of HIV infection. Sex Transm Infect. 2016;92(3):186-193.

66. Ngcapu S, et al. Lower concentrations of chemotactic cytokines and soluble innate factors in the lower female genital tract associated with the use of injectable hormonal contraceptive. J Reprod Immunol. 2015;110:14-21.

67. Nugent RP, Krohn MA, Hillier SL. Reliability of diagnosing bacterial vaginosis is improved by a standardized method of gram stain interpretation. J Clin Microbiol. 1991;29(2):297-301.

68. Egan ME, Lipsky MS. Diagnosis of vaginitis. Am Fam Physician. 2000;62(5):1095-1104. 